\title{
Tokyo Declaration on Research Integrity and Ethical Publication in Science and Medicine in the Asia Pacific Region
}

We, the participants in the Joint Meeting of the Asia Pacific Association of Medical Journal Editors (APAME), the Index Medicus for the South-East Asian Region (IMSEAR), and the Western Pacific Region Index Medicus (WPRIM) held in Tokyo from 2 to 4 August 2013:

\section{CONSIDERING}

That overwhelming data in science and medicine may differ in their reliability and that quality control is important for compiling scientific and health information;

That equitable circulation of scientific and health information is facilitated by fair collaboration among policy makers, researchers, and industry sectors including pharmaceuticals and publishers;

That APAME, IMSEAR, and WPRIM are important collaborative initiatives that can implement global guidelines for publication and dissemination of scientific and medical knowledge in an equitable and ethical manner;

\section{CONFIRM}

Our commitment to endorse that scientific and medical knowledge is imperishable and should not be assessed or evaluated by only economic or temporal considerations;

Our commitment to improve quality and reliability of scientific and medical knowledge through the IMSEAR and WPRIM;

Our commitment to publish reliable and high-quality information by education of researchers, implementation of fair review processes, and organization of networks through APAME;

Our commitment to collaborate with publishers, academic or public libraries, and research bodies to achieve equitable and ethical publication and dissemination of scientific and medical knowledge;

\section{COMMIT}

Ourselves, to publishing reliable and high-quality information, thereby setting the ethical standard for our colleagues, editors, and librarians in the Region;

Our publishers, to disseminating scientific and medical knowledge fairly and impartially through digital library services including, but not limited to, IMSEAR, WPRIM, and the Global Health Library;

Our organization, APAME, to building further networks, convening conferences, and organizing events to educate and empower editors, peer reviewers, and authors to achieve internationally acceptable, but regionally realistic, scholarly standards.

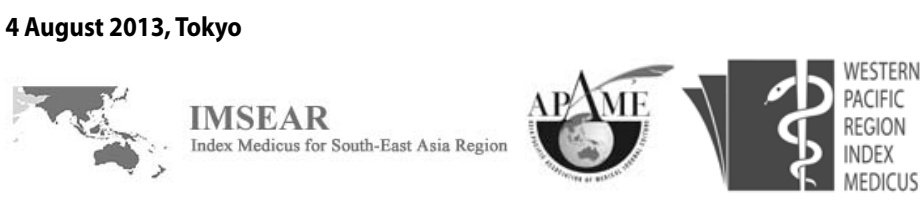

This declaration was adopted at the 2013 Convention of the Asia Pacific Association of Medical Journal Editors (APAME) held in Tokyo from 2 to 4 August 2013. It is concurrently published by Journals linked to APAME and listed in the Index Medicus for the South East Asian Region (IMSEAR) and the Western Pacific Region Index Medicus (WPRIM). Copyright ( $)$ APAME. www.wpro.who.int/apame_apame@wpro.who.int 